\title{
Identification of a new natural gastric lipase inhibitor from star anise
}

3 Jannet Kamoun ${ }^{1,2,3}$, Renaud Rahier ${ }^{3, \S}$, Mohamed Sellami ${ }^{1}$, Imed Koubaa ${ }^{4}$, Pascal Mansuelle ${ }^{5}$,

4 Régine Lebrun ${ }^{5}$, Alexandra Berlioz-Barbier ${ }^{3}$, Karine Alvarez ${ }^{6}$, Abdelkarim Abousalham ${ }^{3}$,

5 Frédéric Carrière ${ }^{2}$, and Ahmed Aloulou, ${ }^{1,{ }^{*}}$

$6{ }^{1}$ University of Sfax, National School of Engineering of Sfax, Laboratory of Biochemistry and $7 \quad$ Enzymatic Engineering of Lipases, Sfax 3038, Tunisia.

$8{ }^{2}$ Aix-Marseille University, CNRS, Bioénergétique et Ingénierie des Protéines, UMR 7281, 9 Marseille 13009, France.

3 Univ. Lyon, Université Lyon 1, Institut de Chimie et de Biochimie Moléculaires et Supramoléculaires (ICBMS), UMR 5246 CNRS, Métabolisme, Enzymes et Mécanismes Moléculaires $\left(\mathrm{MEM}^{2}\right)$, Villeurbanne 69622, France.

${ }^{4}$ University of Sfax, Faculty of Science of Sfax, Laboratory of Chemistry of Natural 14 Products, Sfax 3038, Tunisia.

${ }^{5}$ Aix-Marseille University, CNRS, Institut de Microbiologie de la Méditerranée, Plateforme Protéomique, Formation de Recherche 3479, Marseille 13009, France. Biologiques, UMR 7257, Marseille 13009, France.

$\S$ Present Address: University of Illinois at Chicago, Department of Chemistry, Chicago, Illinois 60607, USA. 


\section{Abbreviations}

24 DGL, dog gastric lipase; HPL, human pancreatic lipase; LipY, mycobacterial Rv3097c25 encoded lipase; SA, star anise; DMSO, dimethyl sulfoxide; M5ME, myricitrin-5-methyl ether; 26 FFA, Free fatty acid; TC4, tributyrin; NaTDC, sodium taurodeoxycholate; SAPRF, star anise 27 polyphenolic-rich fraction; MALDI-TOF-MS; matrix-assisted laser desorption ionization28 time of flight mass spectrometry; PMF, peptide mass fingerprinting; TLC, thin layer 29 chromatography; UPLC-HRMS, ultra-performance liquid chromatography high resolution 30 hybrid quadrupole-time of flight mass spectrometry. 


\section{Abstract}

33 Bioactive compounds identification and isolation from natural complex samples, even though 34 being a difficult task, is of great interest in the drug discovery field. We describe here an innovative strategy for the identification of a new gastric lipase inhibitor from star anise for the treatment of obesity. After plant screening assays for gastric lipase inhibition, star anise was selected and investigated by bioactivity guided fractionation. MALDI-TOF mass spectrometry and peptide mass fingerprinting allowed the detection of an inhibitor covalently bound to the catalytic serine of gastric lipase. Mass-directed screening approach using UPLCHRMS and accurate mass determination searching identified the flavonoid myricitrin-5methyl ether (M5ME) as a lipase inhibitor. The inhibitory activity was rationalized based on molecular docking, showing that M5ME is susceptible to a nucleophilic attack by gastric lipase. Overall, our data suggest that M5ME may be considered as a potential candidate for future application as a lipase inhibitor for the treatment of obesity. Keywords Gastric lipase, inhibition; star anise, guided fractionation; mass spectrometry, molecular 47 docking. 


\section{Introduction}

Gastric and pancreatic lipases play a key role in gastrointestinal digestion of dietary fat suggesting that they may represent a drug target for the treatment of obesity ${ }^{1,2}$. Gastric lipase is the key enzyme for the gastrointestinal digestion of dietary triglycerides ${ }^{3}$. Lipid digestion is initiated in the stomach by the acid stable gastric lipase and continues in the duodenum through the synergistic actions of gastric lipase and colipase-dependent pancreatic lipase, leading to the formation of absorbable monoglycerides and free fatty acids (FFA) ${ }^{4}$. Inhibiting these lipases to reduce fat absorption is the main pharmacological approach adopted for the treatment of obesity ${ }^{5}$. Orlistat, a hydrogenated derivative of lipstatin derived from Streptomyces toxitricini, is the only FDA-approved anti-obesity drug that specifically targets digestive lipases ${ }^{6}$. This drug is an active site-directed inhibitor that reacts with the nucleophilic serine residue of the catalytic triad of digestive lipases within the gastrointestinal tract $^{7}$. However, Orlistat, which is considered relatively too expensive to afford, is also known to inhibit various other enzymes and could lead to undesirable side effects, which might affect its current clinical use ${ }^{8}$. Therefore, looking for new cost-effective digestive lipase inhibitors is highly demanded nowadays.

Much interest has been shifted on plant polyphenols that have been reported to play a significant role in the prevention of overweight and obesity $9,{ }^{10}$. More precisely, polyphenolic-rich extracts from plant sources have been reported to inhibit pancreatic lipase in vitro ${ }^{11-14}$.

Bioactivity guided fractionation is a useful approach for the chemical and biological screening of complex plant extracts ${ }^{15,16}$. Mass spectrometry (MS)-based affinity approaches have emerged as effective tools in small-molecule drug discovery. In these approaches the mass spectrometer can be exploited to rapidly screen drug candidates for specific interactions 
with targets of interest ${ }^{17}$. Coupling bioaffinity selection to MS has been developed in the screening of natural product extracts ${ }^{18}$ and synthetic combinatorial libraries ${ }^{19}$. Accordingly, direct and indirect bioaffinity screening approaches have been used. In the indirect screening approach, the complex protein-ligand is dissociated prior the MS analysis, allowing thus the identification of the target ligand. Whereas, in the direct screening approach, the MS analysis can be directly performed on the whole complex ${ }^{20}$. With the availability of MS technologies, direct bioaffinity screening approach could be a potential way to facilitate tentative identification of the relevant compound within simplified natural mixtures.

UPLC-HRMS is a modern powerful tool to identify known and unknown compounds in a complex mixture. The use of UPLC-HRMS in combination with a QTOF mass spectrometer for target and non-target screening purposes has gained popularity in recent years ${ }^{21}$. Indeed, UPLC provides fast and high-resolution separation, which increases LC-MS sensitivity and minimizes matrix interference arising from minimal simple preparation. Coupling of UPLC system with QTOF-MS provides thus deep analysis on sample composition ${ }^{22}$. Although increasing applications focus on analyzing molecules present in a variety of samples, there have been no reports regarding UPLC-HRMS application for identifying lipase inhibitors from natural product mixture.

To identify a natural gastric lipase inhibitor from plants, we proposed herein a new strategy based on bioactivity guided fractionation along with direct bioaffinity screening using MALDI-TOF-MS and peptide mass fingerprinting (PMF), non-target screening using UPLCHRMS, and in silico molecular docking. 


\section{Materials and methods}

\subsection{Preparation of plant crude extracts for screening study}

Dried plant species, purchased from local markets, were cleaned and finely powdered using a grinder. All powdered samples were separately extracted by maceration technique using hexane, ethyl acetate, ethanol and water $(1: 10 \mathrm{w} / \mathrm{v}$ ratio). The different extracts were evaporated to dryness in vacuo yielding dried extracts. All extracts were dissolved in dimethyl sulfoxide (DMSO) to obtain a final concentration of $100 \mathrm{mg} / \mathrm{mL}$ and were assayed for their anti-lipase potency.

\subsection{Bioactivity guided fractionation}

Star anise crude phenolic extract (SACPE) was prepared from star anise powder via maceration in ethanol:water $(7: 3 \mathrm{v} / \mathrm{v})(1: 10 \mathrm{w} / \mathrm{v}$ ratio) for 24 hours. The solid-liquid mixture was filtered with fritted glass number 3 . Then, the filtrate was evaporated to dryness in vacuo yielding dried SACPE.

Star anise powder was extracted with hexane $(1: 10 \mathrm{w} / \mathrm{v})$ for $48 \mathrm{~h}$ to remove lipoidal material ${ }^{23}$. After filtration, the filtrate was evaporated under vacuum to yield star anise hexanic extract. The residue was air-dried at room temperature overnight to get the dried defatted star anise (DFSA). Thus, star anise phenolic extract (SAPE) was prepared from DFSA via maceration in ethanol:water $(7: 3 \mathrm{v} / \mathrm{v})(1: 10 \mathrm{w} / \mathrm{v}$ ratio $)$ for $24 \mathrm{~h}$.

SAPE was successively fractionated using liquid-liquid partition and solid-liquid extraction to obtain star anise polyphenol-rich fractions (SAPRF). In a first step, liquid-liquid partitioning of SAPE with ethyl acetate gives two fractions SAPRF1 and SAPRF2. These fractions were evaporated under vacuum $\left(45^{\circ} \mathrm{C}\right)$, lyophilized and subjected to the inhibition test against DGL activity. SAPE, SAPRF1 and SAPRF2 were then analyzed by TLC on aluminum sheets coated with $0.2 \mathrm{~mm}$ silica gel 60 to characterize their chemical profile. The migration was performed with a mixture of chloroform:methanol $(8: 2 \mathrm{v} / \mathrm{v})$. After that, the 
spots were revealed with iodine vapor. In a second step, SAPRF2 was re-suspended in ethyl acetate for solid-liquid extraction. After 3 hours of maceration, the mixture was filtered. Similarly, this second step led to two fractions, SAPRF3 and SAPRF4, that were dried under vacuum. Subsequently, SAPRF2 and the fraction produced (SAPRF3 and SAPRF4) were subjected to DGL inhibition test and TLC analysis under the same experimental conditions of the first step. SAPRF4 was thereby selected for further investigations. A flow chart for the preparation of various fractions from star anise powder is given in Fig. 1.

\subsection{Lipase activity measurements using the pH-stat technique}

Recombinant DGL ${ }^{24}$, HPL ${ }^{25}$, and LipY ${ }^{26}$ were produced and purified as described previously. Pancreatic colipase was purified from lipid-free porcine pancreatic powder ${ }^{27}$. Lipases were stored in $150 \mathrm{mM} \mathrm{NaCl}$ buffered solutions (pH 6 for DGL, pH 7 for HPL, and pH 8 for LipY).

Enzymatic activity was assayed at $37^{\circ} \mathrm{C}$ by measuring the amount of FFA released from a mechanically stirred tributyrin (TC4) emulsion, using $0.1 \mathrm{M} \mathrm{NaOH}$ with a pH-stat (Metrohm 718 STAT Titrino, Switzerland) adjusted to a fixed end point ${ }^{28}$. TC4 emulsions were formed by mixing $0.5 \mathrm{~mL}$ TC4 with $14.5 \mathrm{~mL}$ buffer solution. The activities of DGL were determined using the following assay solution: $150 \mathrm{mM} \mathrm{NaCl}, 2 \mathrm{mM}$ sodium taurodeoxycholate (NaTDC), and $2 \mathrm{mM}$ bovine serum albumin at $\mathrm{pH} 5.5^{24}$. In the case of HPL, the activities were determined using the standard assay solution for PL: $0.3 \mathrm{mM}$ Tris$\mathrm{HCl}(\mathrm{pH} 8.0), 150 \mathrm{mM} \mathrm{NaCl}, 2 \mathrm{mM} \mathrm{CaCl}_{2}$, and $4 \mathrm{mM} \mathrm{NaTDC}$, in the presence of 5 molar excess of colipase ${ }^{25}$. With LipY, the assay solution was $2.5 \mathrm{mM}$ Tris- $\mathrm{HCl}(\mathrm{pH} 7.5), 300 \mathrm{mM}$ $\mathrm{NaCl}$ and $3 \mathrm{mM} \mathrm{NaTDC}{ }^{26}$. Under the above assay conditions, enzymatic activities were expressed as international units: $1 \mathrm{U}=1 \mu \mathrm{mol}$ FFA released per minute. The specific activities of DGL, HPL, and LipY, expressed in U per mg of pure enzyme, were found to be $340 \pm 21,8021 \pm 79$ and $129 \pm 2 \mathrm{U} / \mathrm{mg}$, respectively. 


\subsection{Assay for anti-lipase activity}

The lipase-inhibitor pre-incubation method was used to test, in aqueous medium and in the absence of substrate, the possible direct reactions between lipases and inhibitors ${ }^{5}$. Lipaseinhibitor pre-incubations were performed at $25^{\circ} \mathrm{C}$, at different times and at various inhibitor extract amounts $\left(\mathrm{a}_{\mathrm{I}}\right)$, in presence of $4 \mathrm{mM} \mathrm{NaTDC}{ }^{5}$. The final enzyme concentration in the incubation medium was $1 \mu \mathrm{M}$. The residual lipase activity was measured by the $\mathrm{pH}$-stat. The amount of the inhibitor fraction $\left(\mathrm{a}_{150}\right)$ and the half-inactivation time $\left(\mathrm{t}_{1 / 2}\right)$, corresponding to $50 \%$ of residual enzyme activity, were determined.

In each case, control experiments were performed in the absence of inhibitor fraction but with the same volume of DMSO. It is worth noting that DMSO at a final volume concentration less than $10 \%$ has no effect on the enzyme activity.

\subsection{UPLC-HRMS experiment}

The system used for UPLC-HRMS was an ultra-performance liquid chromatography Ultimate 3000 (Thermo Fisher Scientific, Villebon-sur-Yvette, France) coupled to a highresolution hybrid quadrupole-time of flight mass spectrometer (Impact II, Bruker, Brême, Germany) equipped with electrospray ionization source (ESI) (Bruker, Brême Germany). Instrument control and data collection were performed using Data Analysis 5.0 software.

Chromatographic separation was performed on a polar C18 column $(10 \mathrm{~cm} \times 2.1 \mathrm{~mm} \times$ $1.7 \mu \mathrm{m}$, Luna Omega, Phenomenex, France). The column oven temperature was set at $40{ }^{\circ} \mathrm{C}$ and an injection volume of $7 \mu \mathrm{L}$ of the sample was loaded. The sample compounds were separated at a flow rate of $0.4 \mu \mathrm{L} / \min$ using (A) $\mathrm{H}_{2} \mathrm{O}$ (Milli-Q) with $0.1 \%$ formic acid and (B) acetonitrile with $0.1 \%$ formic acid. The analytes were separated using the following gradient: from $0 \%$ to $100 \%$ of $\mathrm{B}$ in $30 \mathrm{~min}$ and kept constant for $5 \mathrm{~min}$. The column was reequilibrated for $10 \mathrm{~min}$ at the initial composition of the gradient before runs. 
The ESI interface was operated on full scan mode $(\mathrm{m} / \mathrm{z} 50-2000)$ in negative and positive ion mode. The parameters for the ESI ion source were as follows: the capillary voltage, $3.0 \mathrm{kV}$; the source temperature, $200{ }^{\circ} \mathrm{C}$; the operating pressure of the nitrogen flow for the nebulizer gas, 45 Psi. Ultraviolet (UV) detectors measured the absorption over the range of 250 and $280 \mathrm{~nm}$.

Before each acquisition batch, external calibration of high-resolution mass spectrometer was performed with a sodium formate cluster solution. The calibration solution was injected at the beginning of each run. MS/MS analyses were carried out with Data Dependent Acquisition mode for precursor ions with an intensity superior to 2000 counts using a stepping of collision energy ramp.

Annotation of each signal was performed by interrogation of different databases (ChemSpider, MassBank, Drugbank, HMDB) using a home-made software providing annotation of LC-MS data according to parent mass accuracy $(<5 \mathrm{ppm})$. After generation of a short list of potential candidates for each signal, the correlation of isotope patterns according to putative atomic compositions was checked to reduce the list of putative annotation (mSigma < 30). The investigation of experimental MS/MS data using web-based spectral database and/or in silico fragmentation tool as MetFrag@ was necessary to obtain structural information and annotate more precisely each compound.

\subsection{MALDI-TOF mass spectrometry analysis}

DGL was pre-incubated for $30 \mathrm{~min}$ at $25^{\circ} \mathrm{C}$ with a large excess of SAPRF4 $\left(\mathrm{a}_{\mathrm{I}}=30 \mu \mathrm{g}\right)$ to abolish any residual lipolytic activity. A blank experiment was performed in the absence of SAPRF4. MALDI-TOF analysis of the entire non-inhibited or inhibited DGL was carried out with a Bruker Micoflex II mass spectrometer (Daltonik, Deutchland) using a saturated solution of $\alpha$-cyano-4-hydroxycinnamic acid in acidified water (0.1\% TFA) and acetonitrile $(30: 70 \mathrm{v} / \mathrm{v})^{28}$. Mass spectra were acquired in the positive ion mode, using the Flex Analysis ${ }^{\mathrm{TM}}$ 
software program (Bruker, Daltonik, Deutchland). Protein identification was performed using the MASCOT ${ }^{\mathrm{TM}}$ version 2.2 search engine (Matrix Science, London, UK) and the NCBI protein database. Theoretical and experimental peptide mass were obtained using the BioTools ${ }^{\mathrm{TM}}$ software program (Bruker, Daltonik, Deutchland).

\subsection{Peptide mass fingerprinting}

Non-inhibited and inhibited DGL (with SAPRF4) were first separated by SDS-PAGE. The protein bands were then excised from the gel and subjected to in-gel trypsin digestion procedure as previously described ${ }^{28}$. For peptides identification, the in-gel digested peptides were analyzed by a proteomic approach including MALDI-TOF/MS and electrospray ionization (ESI) quadrupole time of flight (QTOF) MS/MS (Waters, Manchester) coupled with a nano flow UPLC nano Acquity (Waters, Manchester). For UPLC-ESI-QTOF analysis, the samples were dissolved in the loading buffer (3\% acetonitrile/ $0.1 \%$ TFA in water) and desalted on a C18 nano trap (Symmerty $\mathrm{C} 18,180 \mu \mathrm{m} \times 2 \mathrm{~cm}, 5 \mu \mathrm{m}$, Waters) mounted on a 6port valve, before on line elution onto a C18 column $(\mathrm{BEH} 130 \mathrm{C} 18,100 \mu \mathrm{m} \times 10 \mathrm{~cm}, 1.7$ $\mu \mathrm{m}$, Waters). Gradient elution was performed from $3 \%$ to $50 \%$ of mobile phase B (100\% acetonitrile / $0.1 \%$ formic acid $)$ in $\mathrm{A}(0.1 \%$ formic acid in water) for $30 \mathrm{~min}$. The column was rinsed for 6 min with $85 \%$ of $\mathrm{B}$ and then brought back in 1 min to the initial condition. Between each sample, a blank (injection of loading buffer only) was done using the same chromatographic method. The peptides were detected into the mass spectrometer in a positive ion mode using the $\mathrm{MS}^{\mathrm{E}}$ mode. Data acquisitions of spectra were performed using the Micromass software Protein Lynx Global Server 2.5.2 (Waters).

\subsection{Molecular docking}

In silico molecular docking of the M5ME inhibitor present in the active site of DGL (PDB entry codes: $1 \mathrm{~K} 8 \mathrm{Q}-2.70 \AA$ resolution ${ }^{24}$ ) was performed using AutoDock Vina ${ }^{29}$ program under UCSF Chimera software ${ }^{30}$. The grid box size was chosen to fit the whole 
active site cleft and to allow non-constructive binding positions. The inhibitor structure model was built using the Avogadro program ${ }^{31}$. Binding modes were scored and ranked based on the most favorable energies.

\section{Results}

\subsection{Screening for anti-lipase properties of crude medicinal plant extracts}

Twenty extracts, prepared from five medicinal plants with anti-obesity potential (Illicium verum, Glycyrrhiza glabra, Salvia officinalis, Thymus vulgaris, and Rosmarinus officinalis $)^{32}$, were screened for their inhibitory effect against DGL activity at an ar value of 1 mg. The ethanolic extract of the fruits of Illicium verum (star anise) was found to have the strongest inhibitory activity (81\%) against DGL (Table S1). While the ethanolic extract of Rosmarinus officinalis and the roots of Glycyrrhiza glabra showed a moderate inhibitory activity (43 and $44 \%$, respectively) toward DGL, the remaining medicinal plant extracts displayed weak inhibitory activities. Star anise was thereby selected for further processing.

\subsection{Effect of defatting star anise on anti-lipase activity potency}

Since star anise hexanic extract showed a weak inhibitory rate (18\%, Table S1) and a marked yield (around $7 \%$, data not shown), we evaluated the anti-lipase activity of phenolic extract from star anise before and after defatting sample. Thus, SACPE (non-defatted) and SAPE (defatted) extracts were prepared as described in the experimental section. Using an $a_{I}$ value of $1 \mathrm{mg}$ of each extract, the lipase inhibitory activity of SAPE (100\%) was higher than that of SACPE (89\%).

\subsection{Bioactivity guided fractionation of SAPE}

SAPE was fractionated using the scheme shown in Fig. 1. Two steps were used to fractionate SAPE. In the first step, the fractionation of SAPE led to SAPRF1 and SAPRF2, which were analyzed on TLC (Fig. S1A). One can note that spots intensities in SAPRF1 and 
SAPRF2 have increased compared to the initial extract (SAPE). DGL activity inhibitory test was conducted on each of these two fractions. At an $a_{I}$ value of $500 \mu \mathrm{g}$ of each fraction, SAPRF2 totally abolished DGL residual activity, while SAPRF1 led only to $25 \%$ residual DGL activity. Subsequently, as SAPRF2 fraction was found to be more active, it was further subjected to a second fractionation step. Similarly, this latter led to SAPRF3 and SAPRF4 (Fig. 1) which were subjected to lipase activity inhibitory test and TLC analysis (Fig. S1B). Unlike SAPRF3, SAPRF4 was found to display promising anti-lipase potency with $100 \%$ DGL inhibition rate at an a value of $250 \mu \mathrm{g}$. Regarding TLC analysis, obtained results showed that SAPRF3 is moderately polar fraction, while SAPRF4 seems to have a strong polar character.

\subsection{Evaluation of the inhibitory effect of SAPRF4 against lipases of medicinal interest}

The inhibitory activity of the SAPRF4 fraction was evaluated against two mammalian digestive lipases (DGL and HPL) and one microbial lipase belonging to the hormonesensitive lipase (HSL) family (LipY) ${ }^{26}$. With each lipase, linear kinetics corresponding to the FFAs $(\mu \mathrm{mol})$ released $v s$. time (min) were obtained in the presence and absence of the inhibitor fraction. A dose-dependent effect was observed upon increasing the amount of SAPRF4 on DGL, HPL and LipY (Fig. 2). At an a value of only $30 \mu \mathrm{g}$, DGL activity was totally abolished after a 30 min incubation period (Fig. 2A), while HPL was strongly inactivated $(96 \%)$ at an $a_{I}$ value of up to $1 \mathrm{mg}$ (Fig. 2B). However, LipY activity was not completely abolished even at a high SAPRF4 amount, showing a residual activity of $\sim 20 \%$ (Fig. 2C). The $\mathrm{a}_{150}$ values were found to be $7 \mu \mathrm{g}, 82 \mu \mathrm{g}$ and $5 \mu \mathrm{g}$ for DGL, HPL and LipY, respectively (Table 1), indicating that SAPRF4 is a potent inhibitor toward both DGL and LipY.

The influence of the incubation time on the level of inhibition of DGL, HPL and LipY by the SAPRF4 fraction was further investigated (Fig. 2D-F). The residual activity of these 
lipases decreased rapidly and reached a plateau value after approximately $30 \mathrm{~min}$ of incubation. From these inhibition curves, values of the half-inactivation times $\left(t_{1 / 2}\right)$ were then determined and found to be $0.4 \mathrm{~min}, 0.6 \mathrm{~min}$ and $0.1 \mathrm{~min}$ for DGL, HPL and LipY, respectively (Table 1). Such values reflect an extremely high rate of inhibition of these lipases by SAPRF4.

\subsection{Analysis of the inhibitor-modified DGL complex with mass spectrometry}

To investigate whether the inhibitory compound within SAPRF4 forms a covalent bond with the catalytic serine of DGL, MALDI-TOF-MS analysis was conducted on both noninhibited and inhibited DGL. Fig. 3 shows typical spectral recordings of untreated and treated DGL with SAPRF4. A clear shift in the molecular mass of DGL was observed reflecting a covalent binding of the inhibitor to the lipase.

To deduce the exact molecular mass of the inhibitor contained in the SAPRF4 fraction, PMF analysis of non-inhibited and inhibited DGL was performed. Results showed that the peptide $\mathrm{L}^{147}-\mathrm{K}^{168}$ (LHYVGHSQGTTIGFIAFSTNPK) containing the catalytic $\mathrm{S}^{153}$ residue was detected at a molecular mass of 2376.05 Da with non-inhibited DGL (Fig. 4A). This catalytic peptide appeared concomitantly with a second peptide detected at a molar mass of around 2414.82 Da. By contrast, when DGL was incubated with SAPRF4, a mass increase of + 489.52 Da was observed in the PMF spectrum for the catalytic peptide (Fig. 4B), while the peptide detected at a molar mass of around 2414.82 Da remained unchanged. This mass shift (489.52 Da) observed here is supposed to correspond to the molecular mass of the natural inhibitor bound to DGL.

To tentatively identify the molecular formula and the structure of the phytochemical compound that binds covalently to DGL, we analyzed the SAPRF4 fraction by UPLC-HRMS. Seven major phenolic compounds have been identified (Fig. S2A and Table S2). To empirically calculate their potential molecular formulas, various criteria have been used 
among them: the isotopic pattern, the fragmentation pattern and the high-resolution accurate mass (with error below $0.4 \mathrm{ppm}$ at the time of matching the theoretical mass with the measured mass). Mass-directed identification showed that the natural DGL inhibitor compound within the SAPRF4 fraction might correspond to myricitrin-5-methyl ether (M5ME) with a molecular mass of $479.1184 \mathrm{Da}$ and a molecular formula of $\mathrm{C}_{22} \mathrm{H}_{22} \mathrm{O}_{12}$ (Fig. 5).

\subsection{Molecular docking}

To shed light on DGL-M5ME binding interactions, in silico molecular docking was performed using AutoDock Vina program. The open conformation of DGL, in complex with a phosphonate inhibitor covalently bound to its catalytic serine residue ${ }^{24}$, was used as reference model to improve the reliability of docking results. The lipase active site was found to be fully accessible thus enabling docking experiments. Automated docking resulted in several possible conformations of the M5ME inhibitor within DGL catalytic pocket, with favorable binding energies ranging from -8.3 to $-9.5 \mathrm{kcal} / \mathrm{mol}$ (Fig. S3A). For subsequent analysis, we retained the best matching conformation that directly exposes the most reactive carbon atom of the M5ME inhibitor towards the catalytic serine, within a calculated distance of $3.8 \AA$ which is sufficient to promote the nucleophilic attack (Fig. S3B). In this configuration, we found that M5ME could fit properly into DGL catalytic pocket (Fig. 6), which consists of a deep canyon of approximately $20 \AA$ long, $7 \AA$ wide, and $20 \AA$ deep ${ }^{24}$. Moreover, like the phosphonate inhibitor, M5ME shows a relatively flat structure, with measured lengths of around $12 \AA$ long, $5.5 \AA$ wide, and $9 \AA$ deep (Fig. S3C). For the sake of comparison, the previously reported phosphonate inhibitor only measures $6 \AA$ long, $2 \AA$ wide and $10 \AA$ deep, and thus requires the presence of $\beta$-octyl glucoside or 1-amino-anthracene to completely fill the catalytic pocket ${ }^{24}$. Interestingly, a more detailed analysis of the environment of the M5ME compound, bond to the catalytic serine, revealed a significant stabilizing effect of the DGL-M5ME tetrahedral 
intermediate by the neighboring oxyanion hole (Fig. 5). Indeed, an oxygen originating from the pyran ring of M5ME points towards the backbone nitrogen atoms of Leu67 and Gln154 residues within hydrogen bond distances of $2.37 \AA$ and $2.08 \AA$, respectively.

\section{Discussion}

Star anise (Illicium verum) is an important traditional Chinese medicine as well as a commonly used spice. Its tree is an aromatic evergreen tree growing almost exclusively in southern China and Vietnam. The fruit of star anise is used in traditional medicine to treat stomachache, colic, vomiting, insomnia, skin inflammation, rheumatic pain, dyspepsia, facial paralysis, asthma, and bronchitis ${ }^{33}$. A great deal of research effort is being devoted to testing the putative beneficial effects of star anise extracts. Modern pharmacology studies demonstrated that crude extracts and active compounds of star anise possess wide pharmacological actions ${ }^{34}$. However, to the best of our knowledge, none has yet examined the effect of star anise extracts on digestive lipases activities.

DGL provides a good model for human gastric lipases ${ }^{24,35}$. A screening of a collection of 20 extracts from five medicinal plants using solvents with different polarities allowed the identification of ethanolic extract from star anise, which efficiently inhibited gastric lipase (Table S1). The inhibitory effect of star anise ethanolic extract on DGL might be caused by the presence of phenolic compounds within the extract. It was reported that ethanol/water extracts of star anise contained high amounts of phenolic compounds ${ }^{36}$. The use of water in combination with ethanol contributes to the creation of a moderately polar medium that ensures the extraction of phenolic compounds ${ }^{37}$. Plant phenolic compounds, such as flavones, flavanols, tannins and chalcones, have been reported as pancreatic lipase inhibitors ${ }^{14}$, thus supporting the use of star anise polyphenolic-rich extract for further bioactivity guided fractionation (Fig. 1). 
Our results showed that removal of lipoidal material from star anise before phenolic extraction enhanced anti-lipase activity, which might be related to the fact that the concentration of phenolics in SACPE is lower than in SAPE, suggesting thereby that the antilipase effect is related to star anise phenolic compounds. One of the hallmarks of bioactivity guided fractionation is the trend of increasing potency with increasing fractionation. Indeed, the $a_{I}$ value that totally abolishes gastric lipase activity moved from $1 \mathrm{mg}$ SAPE to $30 \mu \mathrm{g}$ SAPRF4 after two fractionation steps. According to TLC analysis, SAPRF4 produced by this fractionation approach is a less complex mixture than the initial SAPE (Fig. S1). We therefore sought to evaluate SAPRF4 potency and selectivity against other lipases of medicinal interest. Inhibiting lipases has potential applications in the field of medicine. The digestive lipase inhibitor Orlistat used for the treatment of obesity has been shown to also inhibit HSL ${ }^{38}$ and microbial lipases ${ }^{8,39}$. In the sake of comparison, SAPRF4 was found to inhibit efficiently DGL, HPL, and LipY (a microbial lipase belonging to the HSL family). The ability of SAPRF4 to inhibit the catalytic activity of these lipases was assessed in terms of $a_{150}$ and $t_{1 / 2}$ values (Table 1). The inhibition of HSL could be a potential approach to reduce levels of circulating FFAs linked to insulin resistance in obese patients ${ }^{40}$. Consequently, SAPRF4 might be an effective candidate for the treatment of obesity and diabetes. Moreover, as LipY contributes to the growth and pathogenicity of $M$. tuberculosis ${ }^{26}$, it would be a therapeutic target for SAPRF4 as an anti-microbial agent.

On the bioaffinity interaction level, inhibitors can bind either covalently or noncovalently with their biological target ${ }^{41}$. In the cases of non-covalent inhibition, the inhibitor 361 does not bind covalently with the biological target. Thereby, non-covalent inhibitor compounds are of no special interest in drug discovery projects ${ }^{16}$. MALDI-TOF mass spectrometry is an analytical instrument tool that can measure protein-inhibitor complex mass when a specific covalent binding occurs, reflecting thereby an approximate molecular mass of 
the inhibitor ${ }^{38}$. Direct MALDI-TOF mass spectrometry analysis of DGL totally inhibited by SAPRF4 showed the occurrence of an increase in the molecular masses of DGL, which is compatible with the formation of a covalent complex with the inhibitor. PMF analysis of a trypsin digest of DGL inhibited or not with SAPRF4 showed an increase in the molecular mass of the catalytic serine-containing peptide (Fig. 4) corresponding in size to the approximate molecular mass of the inhibitor. This process might involve a nucleophilic attack between the enzyme's catalytic serine and the inhibitor, leading to the formation of a covalent inhibitor-modified enzyme. Based on PMF analysis, it has been also shown previously that synthetic inhibitors, oxadiazolones ${ }^{28}$ and cyclophostin analogs ${ }^{8,42}$, form a covalent bond with the catalytic serine residue of lipases. To the best of our knowledge, this is the first study that used covalent inhibitor-modified enzyme complex data to tentatively identify a lipase inhibitor in a natural extract.

Achieving the approximate molecular mass of the covalent lipase inhibitor, the identification of the compound of interest within SAPRF4 would be rather more straightforward. Thus, using the inhibitor mass information, we applied UPLC-HRMS for mass-directed screening to tentatively identify the expected lipase inhibitor in SAPRF4. Using this non-target screening, it was possible to separate and detect seven chromatographic peaks containing high resolution MS information (Fig. S2). The tentative identification of these peaks was performed according to their high-resolution accurate mass and isotopic patterns to empirically determine their molecular formulas. By inspecting all mass spectra around $\mathrm{m} / \mathrm{z}$ of 489.52 in the chromatogram of SAPRF4, one ion having $\mathrm{m} / \mathrm{z}$ of 479.1187 was found to fit with PMF analysis data. This ion, assigned to the molecular formula $\mathrm{C}_{22} \mathrm{H}_{22} \mathrm{O}_{12}$, corresponds likely to the flavonoid myricitrin-5-methyl ether (Fig. 5) that has not been identified previously from star anise. 
To gain insights into the lipase-inhibitor binding interactions at the molecular level, M5ME was docked into the active site of DGL (Fig. 6). The final selection of M5ME "bioactive" conformation suggests that the inhibition mechanism involve a nucleophilic attack by the hydroxy group of the catalytic Ser on a reactive carbon atom of the inhibitor pyran ring, thus leading to the formation of a stoichiometric enzyme-inhibitor covalent complex

(Fig. 5). Interestingly, the nucleophilic attack could be favored by some surrounding residues, including the backbone nitrogen atoms of $\mathrm{Leu}^{67}$ and $\mathrm{Gln}^{154}$ that belong to the previously described DGL oxyanion hole ${ }^{24}$.

Much more interest has been shifted on plant flavonoids for their possible anti-obesity effects. In vitro and in vivo studies provided evidence on the potential role that flavonoids play in the management of obesity ${ }^{43}$. M5ME, a myricetin glycoside, was isolated from the flower of Rhododendron poukhanense as a potent antioxidant flavonoid. Although no lipase inhibiting activity was reported in the literature for M5ME, the structurally fairly similar quercetin and kaemferol were reported to be potent pancreatic lipase inhibitors ${ }^{44}$. Moreover, myricetin was found to exert in vivo a strong anti-obesity and anti-hyperlipidaemic activities by modulating the lipid metabolism ${ }^{45}$.

To our knowledge, this is the first report that tentatively identified a new natural gastric lipase inhibitor from a natural product. The combination of in silico molecular docking results with those of lipase inhibition and mass spectrometry provides a scientific evidence for the potential medicinal use of the flavonoid myricitrin-5-methyl ether as a lipase inhibitor for the treatment of obesity, type 2 diabetes and tuberculosis. 


\section{Author contributions}

J.K. carried out experiments and performed analyses. R.R., M.S., P.M., R.L. and A.B.-B. contributed to experiments realization and analysis. I.K., K.A., A.A. and F.C. contributed to experiments design and analysis. J.K. and A.A. conceived experiments and wrote the paper.

\section{Declaration of interests}

There are no conflicts of interest to declare.

\section{Acknowledgments}

This work was supported by the Ministry of Higher Education and Scientific Research, Tunisia. Dr. Stéphane Canaan and Dr. Jean-François Cavalier (CNRS, Marseille) are acknowledged for their generous gift of LipY enzyme and for useful discussions.

\section{References}

1. A. Aloulou and F. Carriere, Gastric lipase: an extremophilic interfacial enzyme with medical applications, Cell Mol Life Sci, 2008, 65, 851-854.

2. A. Aloulou, D. Puccinelli, J. Sarles, R. Laugier, Y. Leblond and F. Carriere, In vitro comparative study of three pancreatic enzyme preparations: dissolution profiles, active enzyme release and acid stability, Aliment Pharmacol Ther, 2008, 27, 283-292.

3. F. Carriere, E. Rogalska, C. Cudrey, F. Ferrato, R. Laugier and R. Verger, In vivo and in vitro studies on the stereoselective hydrolysis of tri- and diglycerides by gastric and pancreatic lipases, Bioorganic \& medicinal chemistry, 1997, 5, 429-435.

4. F. Carriere, J. A. Barrowman, R. Verger and R. Laugier, Secretion and contribution to lipolysis of gastric and pancreatic lipases during a test meal in humans, Gastroenterology, 1993, 105, 876-888. 
5. H. Lengsfeld, Physiology of Gastrointestinal Lipolysis and Therapeutical Use of Lipases and Digestive Lipase Inhibitors, Lipases and Phospholipases in Drug Development: From Biochemistry to Molecular Pharmacology, 2005, DOI: 10.1002/3527601910.ch10, 195-223.

6. H. L. Daneschvar, M. D. Aronson and G. W. Smetana, FDA-Approved Anti-Obesity Drugs in the United States, Am J Med, 2016, 129, 879 e871-876.

7. F. Carriere, C. Renou, S. Ransac, V. Lopez, J. De Caro, F. Ferrato, A. De Caro, A. Fleury, P. Sanwald-Ducray, H. Lengsfeld, C. Beglinger, P. Hadvary, R. Verger and R. Laugier, Inhibition of gastrointestinal lipolysis by Orlistat during digestion of test meals in healthy volunteers, American journal of physiology. Gastrointestinal and liver physiology, 2001, 281, G16-28.

8. V. Point, R. K. Malla, S. Diomande, B. P. Martin, V. Delorme, F. Carriere, S. Canaan, N. P. Rath, C. D. Spilling and J. F. Cavalier, Synthesis and kinetic evaluation of cyclophostin and cyclipostins phosphonate analogs as selective and potent inhibitors of microbial lipases, J Med Chem, 2012, 55, 10204-10219.

9. L. Titta, M. Trinei, M. Stendardo, I. Berniakovich, K. Petroni, C. Tonelli, P. Riso, M. Porrini, S. Minucci, P. G. Pelicci, P. Rapisarda, G. Reforgiato Recupero and M. Giorgio, Blood orange juice inhibits fat accumulation in mice, International journal of obesity, 2010, 34, 578-588.

10. R. B. Birari, S. Gupta, C. G. Mohan and K. K. Bhutani, Antiobesity and lipid lowering effects of Glycyrrhiza chalcones: experimental and computational studies, Phytomedicine : international journal of phytotherapy and phytopharmacology, 2011, 18, 795-801. 
11. Y. Gu, W. J. Hurst, D. A. Stuart and J. D. Lambert, Inhibition of key digestive enzymes by cocoa extracts and procyanidins, Journal of agricultural and food chemistry, 2011, 59, 5305-5311.

12. M. Yoshikawa, H. Shimoda, N. Nishida, M. Takada and H. Matsuda, Salacia reticulata and its polyphenolic constituents with lipase inhibitory and lipolytic activities have mild antiobesity effects in rats, The Journal of nutrition, 2002, 132, 1819-1824.

13. N. Yuda, M. Tanaka, M. Suzuki, Y. Asano, H. Ochi and K. Iwatsuki, Polyphenols extracted from black tea (Camellia sinensis) residue by hot-compressed water and their inhibitory effect on pancreatic lipase in vitro, Journal of food science, 2012, 77, H254-261.

14. R. B. Birari and K. K. Bhutani, Pancreatic lipase inhibitors from natural sources: unexplored potential, Drug discovery today, 2007, 12, 879-889.

15. F. E. Koehn and G. T. Carter, The evolving role of natural products in drug discovery, Nat Rev Drug Discov, 2005, 4, 206-220.

16. M. G. Weller, A unifying review of bioassay-guided fractionation, effect-directed analysis and related techniques, Sensors (Basel), 2012, 12, 9181-9209.

17. S. A. Hofstadler and K. A. Sannes-Lowery, Applications of ESI-MS in drug discovery: interrogation of noncovalent complexes, Nat Rev Drug Discov, 2006, 5, $585-595$.

18. L. L. Cummins, S. Chen, L. B. Blyn, K. A. Sannes-Lowery, J. J. Drader, R. H. Griffey and S. A. Hofstadler, Multitarget affinity/specificity screening of natural products: finding and characterizing high-affinity ligands from complex mixtures by using highperformance mass spectrometry, J Nat Prod, 2003, 66, 1186-1190.

19. B. M. Johnson, D. Nikolic and R. B. van Breemen, Applications of pulsed ultrafiltration-mass spectrometry, Mass Spectrom Rev, 2002, 21, 76-86. 
20. H. Vu, N. B. Pham and R. J. Quinn, Direct screening of natural product extracts using mass spectrometry, J Biomol Screen, 2008, 13, 265-275.

21. D. C. Hilton, R. S. Jones and A. Sjodin, A method for rapid, non-targeted screening for environmental contaminants in household dust, J Chromatogr A, 2010, 1217, 6851-6856.

22. M. Ibáñez, J. V. Sancho, F. Hernández, D. McMillan and R. Rao, Rapid non-target screening of organic pollutants in water by ultraperformance liquid chromatography coupled to time-of-light mass spectrometry, TrAC Trends in Analytical Chemistry, 2008, 27, 481-489.

23. M. A. Ramirez-Coronel, N. Marnet, V. S. Kolli, S. Roussos, S. Guyot and C. Augur, Characterization and estimation of proanthocyanidins and other phenolics in coffee pulp (Coffea arabica) by thiolysis-high-performance liquid chromatography, Journal of agricultural and food chemistry, 2004, 52, 1344-1349.

24. A. Roussel, N. Miled, L. Berti-Dupuis, M. Riviere, S. Spinelli, P. Berna, V. Gruber, R. Verger and C. Cambillau, Crystal structure of the open form of dog gastric lipase in complex with a phosphonate inhibitor, J Biol Chem, 2002, 277, 2266-2274.

25. V. Belle, A. Fournel, M. Woudstra, S. Ranaldi, F. Prieri, V. Thome, J. Currault, R. Verger, B. Guigliarelli and F. Carriere, Probing the opening of the pancreatic lipase lid using site-directed spin labeling and EPR spectroscopy, Biochemistry, 2007, 46, 22052214.

26. P. Santucci, S. Diomande, I. Poncin, L. Alibaud, A. Viljoen, L. Kremer, C. de Chastellier and S. Canaan, Delineating the physiological roles of the PE and catalytic domain of LipY in lipid consumption in mycobacteria-infected foamy macrophages, Infect Immun, 2018, DOI: 10.1128/IAI.00394-18. 
27. C. Chapus, P. Desnuelle and E. Foglizzo, Stabilization of the C-terminal part of pig and horse colipase by carboxypeptidase and trypsin inhibitors, Eur J Biochem, 1981, 115, 99-105.

28. V. Point, K. V. Pavan Kumar, S. Marc, V. Delorme, G. Parsiegla, S. Amara, F. Carriere, G. Buono, F. Fotiadu, S. Canaan, J. Leclaire and J. F. Cavalier, Analysis of the discriminative inhibition of mammalian digestive lipases by 3-phenyl substituted 1,3,4-oxadiazol-2(3H)-ones, Eur J Med Chem, 2012, 58, 452-463.

29. O. Trott and A. J. Olson, AutoDock Vina: improving the speed and accuracy of docking with a new scoring function, efficient optimization, and multithreading, $J$ Comput Chem, 2010, 31, 455-461.

30. E. F. Pettersen, T. D. Goddard, C. C. Huang, G. S. Couch, D. M. Greenblatt, E. C. Meng and T. E. Ferrin, UCSF Chimera--a visualization system for exploratory research and analysis, J Comput Chem, 2004, 25, 1605-1612.

31. M. D. Hanwell, D. E. Curtis, D. C. Lonie, T. Vandermeersch, E. Zurek and G. R. Hutchison, Avogadro: an advanced semantic chemical editor, visualization, and analysis platform, J Cheminform, 2012, 4, 17.

32. J.-C. Charrié, B. Chastel, C. Cieur, P. Combe, M. Damak, K. Hedayat and C. SaigneSoulard, Plantes médicinales - Phytothérapie clinique intégrative et médecine endobiogénique, Lavoisier S.A.S., 2017.

33. Y. Y. Sung, W. K. Yang, A. Y. Lee, D. S. Kim, K. J. Nho, Y. S. Kim and H. K. Kim, Topical application of an ethanol extract prepared from Illicium verum suppresses atopic dermatitis in NC/Nga mice, J Ethnopharmacol, 2012, 144, 151-159.

34. G. W. Wang, W. T. Hu, B. K. Huang and L. P. Qin, Illicium verum: a review on its botany, traditional use, chemistry and pharmacology, J Ethnopharmacol, 2011, 136, $10-20$. 
35. S. Fernandez, S. Chevrier, N. Ritter, B. Mahler, F. Demarne, F. Carriere and V. Jannin, In vitro gastrointestinal lipolysis of four formulations of piroxicam and cinnarizine with the self emulsifying excipients Labrasol and Gelucire 44/14, Pharm Res, 2009, 26, 1901-1910.

36. A. Padmashree, N. Roopa, A. D. Semwal, G. K. Sharma, G. Agathian and A. S. Bawa, Star-anise (Illicium verum) and black caraway (Carum nigrum) as natural antioxidants, Food Chemistry, 2007, 104, 59-66.

37. C. Liyana-Pathirana and F. Shahidi, Optimization of extraction of phenolic compounds from wheat using response surface methodology, Food Chemistry, 2005, 93, 47-56.

38. Y. Ben Ali, H. Chahinian, S. Petry, G. Muller, R. Lebrun, R. Verger, F. Carriere, L. Mandrich, M. Rossi, G. Manco, L. Sarda and A. Abousalham, Use of an inhibitor to identify members of the hormone-sensitive lipase family, Biochemistry, 2006, 45, 14183-14191.

39. R. Dhouib, A. Ducret, P. Hubert, F. Carriere, S. Dukan and S. Canaan, Watching intracellular lipolysis in mycobacteria using time lapse fluorescence microscopy, Biochim Biophys Acta, 2011, 1811, 234-241.

40. G. Boden, Interaction between free fatty acids and glucose metabolism, Curr Opin Clin Nutr Metab Care, 2002, 5, 545-549.

41. H. Gohlke and G. Klebe, Approaches to the description and prediction of the binding affinity of small-molecule ligands to macromolecular receptors, Angew Chem Int Ed Engl, 2002, 41, 2644-2676.

42. V. Point, R. K. Malla, F. Carriere, S. Canaan, C. D. Spilling and J. F. Cavalier, Enantioselective inhibition of microbial lipolytic enzymes by nonracemic monocyclic enolphosphonate analogues of cyclophostin, J Med Chem, 2013, 56, 4393-4401. 
555 43. N. Al Shukor, K. Raes, G. Smagghe and J. Van Camp, in Flavonoids and antioxidants, Studium Press LLC, New Delhi, India, 2016, vol. 40, pp. 496-514.

557

558

559

560

561

562

563

44. T. Sergent, J. Vanderstraeten, J. Winand, P. Beguin and Y.-J. Schneider, Phenolic compounds and plant extracts as potential natural anti-obesity substances, Food Chemistry, 2012, 135, 68-73.

45. C. J. Chang, T. F. Tzeng, S. S. Liou, Y. S. Chang and I. M. Liu, Myricetin Increases Hepatic Peroxisome Proliferator-Activated Receptor alpha Protein Expression and Decreases Plasma Lipids and Adiposity in Rats, Evid Based Complement Alternat Med, 2012, 2012, 787152. 
FIGURE LEGENDS

566 Fig. 1. Bioactivity-guided fractionation scheme of star anise.

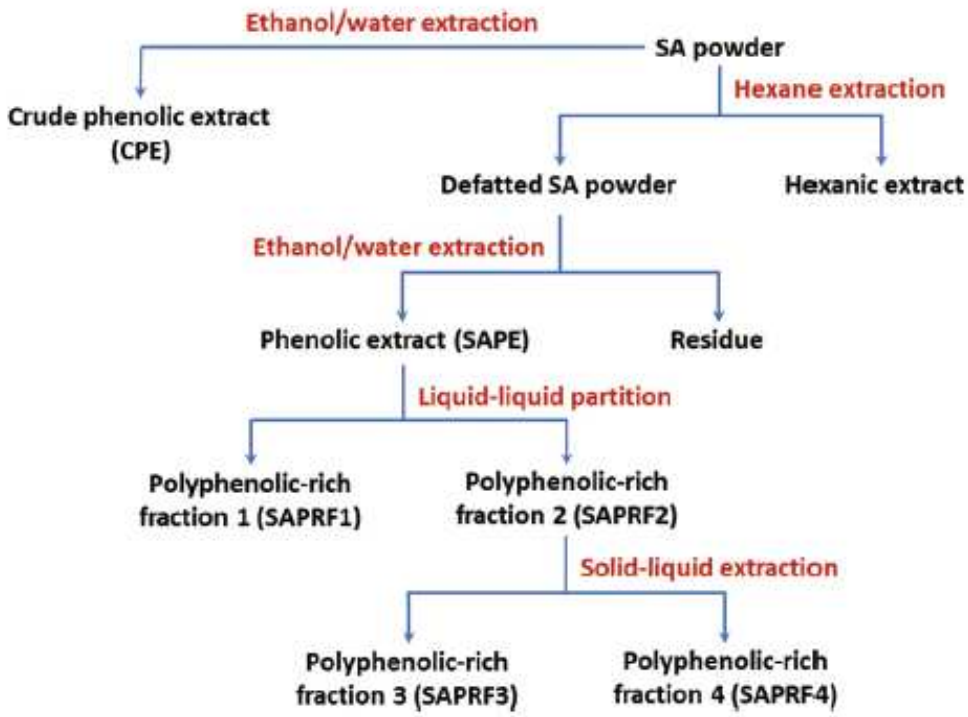


569 LipY lipases. Each lipase was pre-incubated in the presence of various SAPRF4 amounts for

57030 min at $25{ }^{\circ} \mathrm{C}$ and residual activities of DGL (A), HPL (B) and LipY (C) were measured.

571 Alternatively, residual activities of DGL (D), HPL (E) and LipY (F) were measured as a

572 function of the incubation time at a constant SAPRF4 amount. The $a_{I}$ value used in these latter

573 experiments was chosen so that the average residual activities of DGL, HPL and LipY

574 obtained after 30 min of incubation were in the 15-20\% range. Results are expressed as mean

575 values of at least three independent assays.
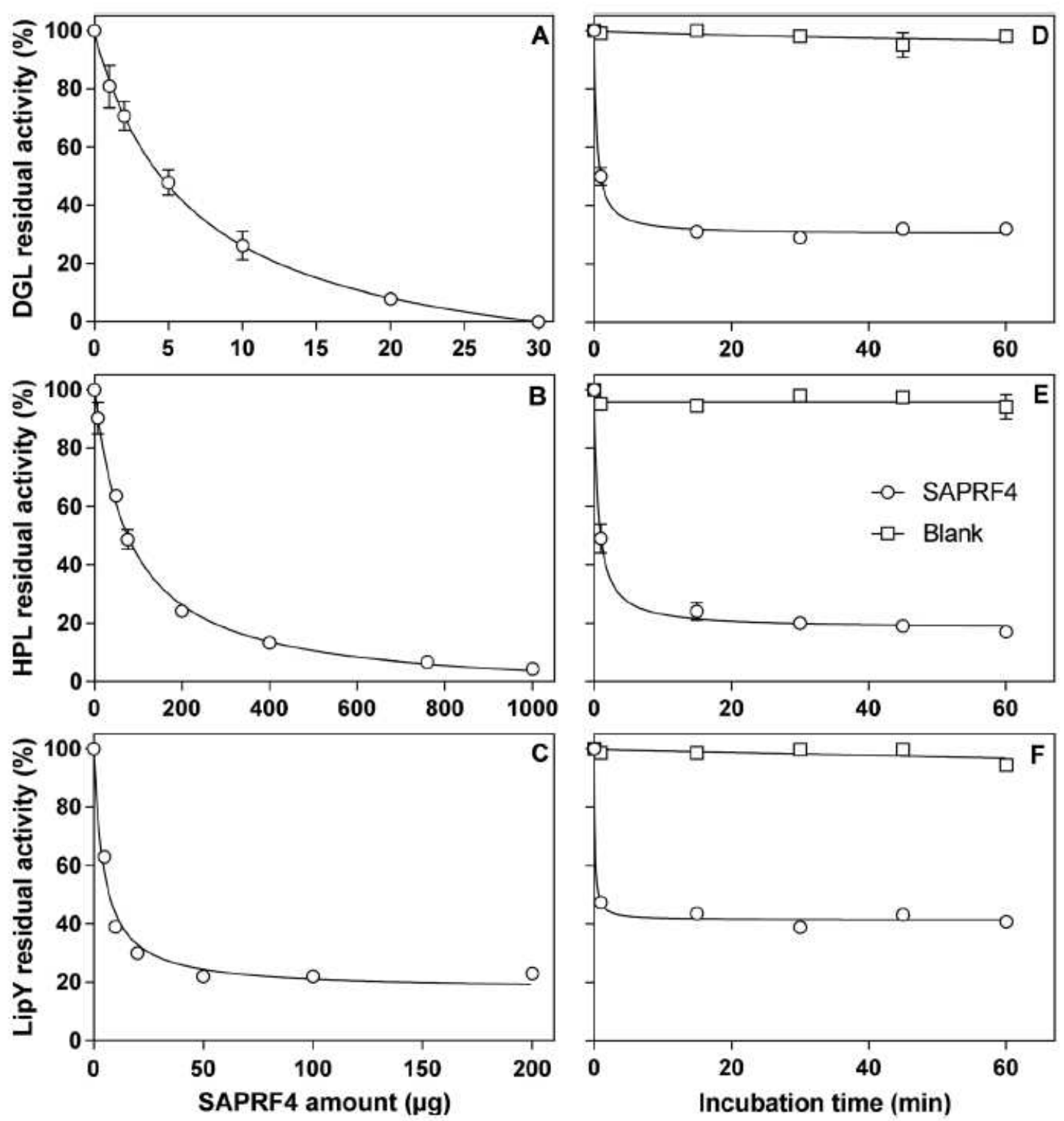
577 Fig. 3. MALDI-TOF mass spectrometry analysis of untreated (grey spectrum) and

578 treated (black spectrum) DGL. A 30-min incubation was performed at $25^{\circ} \mathrm{C}$ in the 579 presence or absence of SAPRF4 amount of $30 \mu \mathrm{g}$.

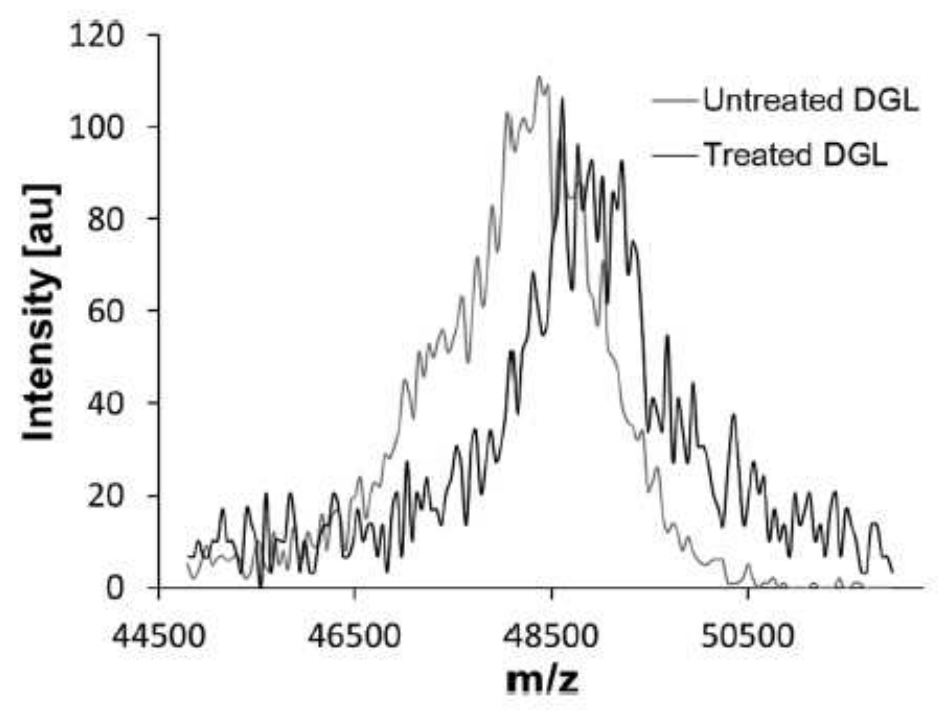


582 performed at $25^{\circ} \mathrm{C}$ in the presence or absence of SAPRF4 amount of $30 \mu \mathrm{g}$. Left panels,

583 region of the unmodified isotopic peptide $\mathrm{L}^{147}-\mathrm{K}^{168}$ containing the catalytic Ser ${ }^{153}$ and 584 detected at 2376.05 Da. This peak is overlaid with a second one corresponding to the peptide 585 detected at 2414.82 Da. Right panels, region in which a new isotopic peptide was expected to 586 occur, resulting from the covalent binding of the natural inhibitor of interest to the catalytic 587 serine. Mass shift was calculated as the difference between the experimental $\mathrm{m} / \mathrm{z}$ of the 588 unmodified peptide and the theorical $\mathrm{m} / \mathrm{z}$ of the unmodified peptide.

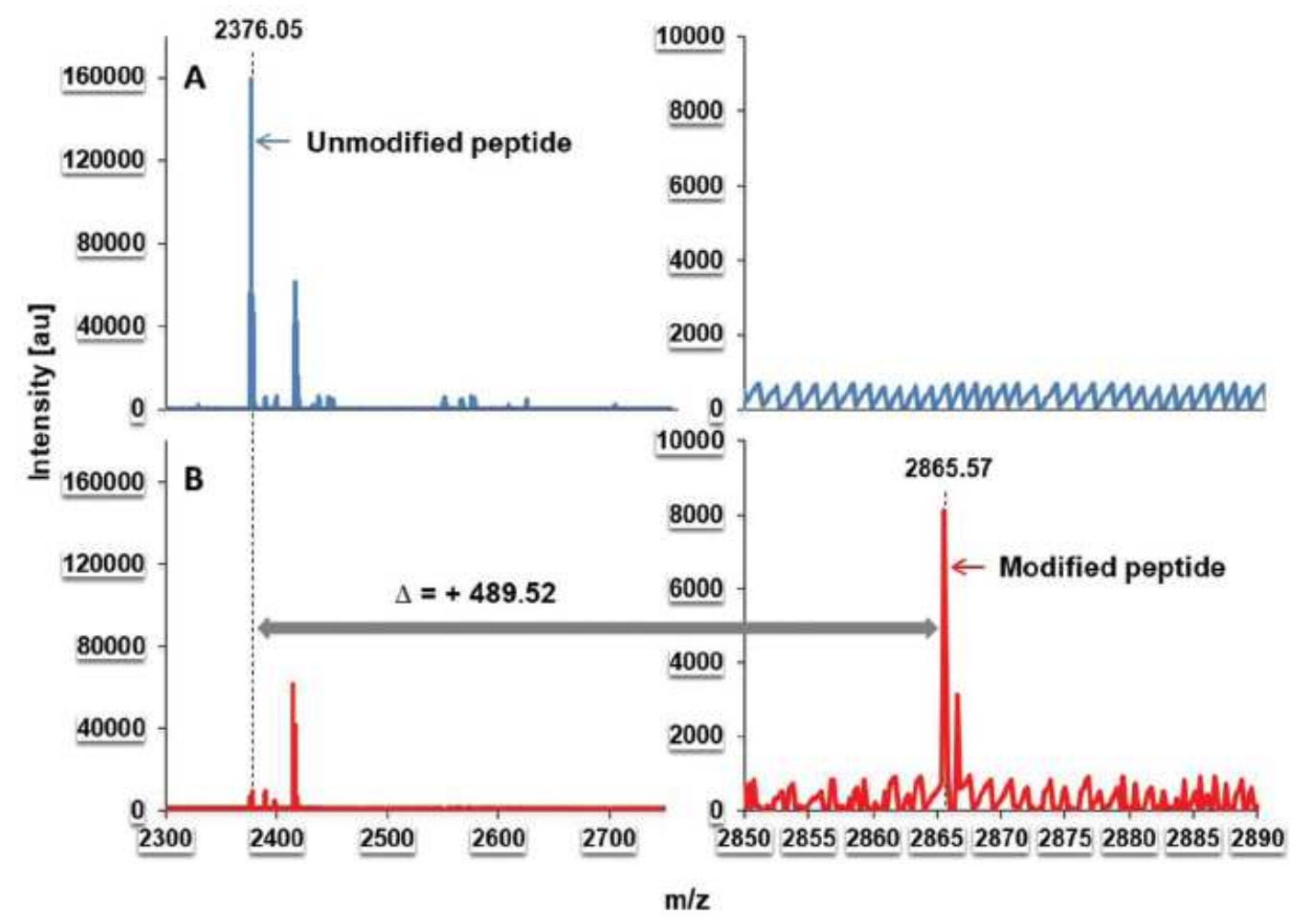


591 proposed mechanism underlying DGL inhibition by M5ME. The nucleophilic attack by

592 the hydroxyl group of the catalytic $\operatorname{Ser}^{153}$ residue on the inhibitor reactive site leads to a 1,4-

593 nucleophilic addition on a double bond conjugated to a ketone and the formation of an enol 594 function.

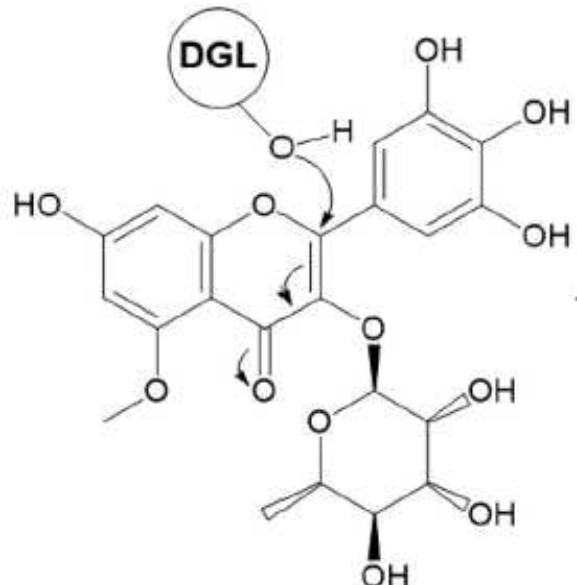

Nucleophilic conjugate addition<smiles>C=C1O[C@H](OC2=C(O)c3c(OC)cc(O)cc3OC2(Oc2ccccc2)c2cc(O)c(O)c(O)c2)C(=O)[C@@H](O)[C@@H]1O</smiles> 
597 molecular surface representation of DGL active site crevice with the bound M5ME. B, view

598 of the active site of DGL with the bound M5ME. The backbone nitrogens of residues Leu ${ }^{67}$ 599 and $\mathrm{Gln}^{154}$ are at hydrogen bonding distance from an oxygen atom originating from the 600 inhibitor. The catalytic residues $\mathrm{Ser}^{153}, \mathrm{His}^{353}$ and $\mathrm{Asp}^{324}$ are highlighted in pink and the 601 inhibitor is represented in CPK coloring (oxygen, red).
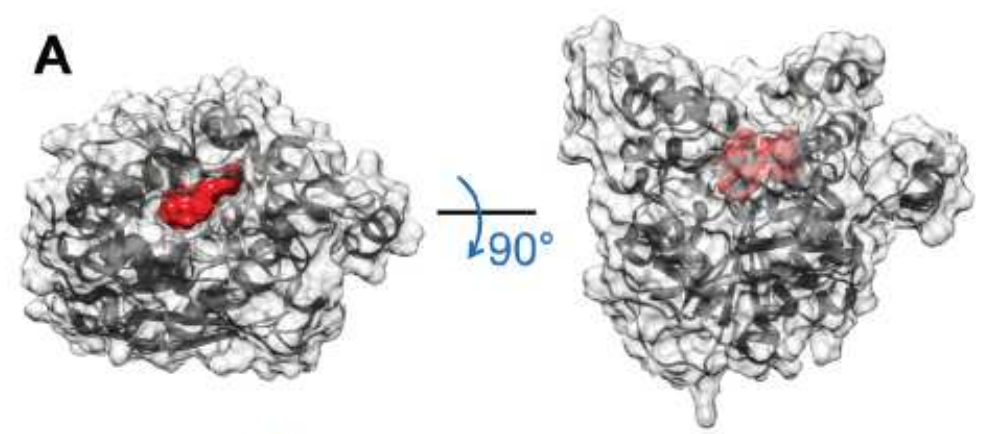

B

602

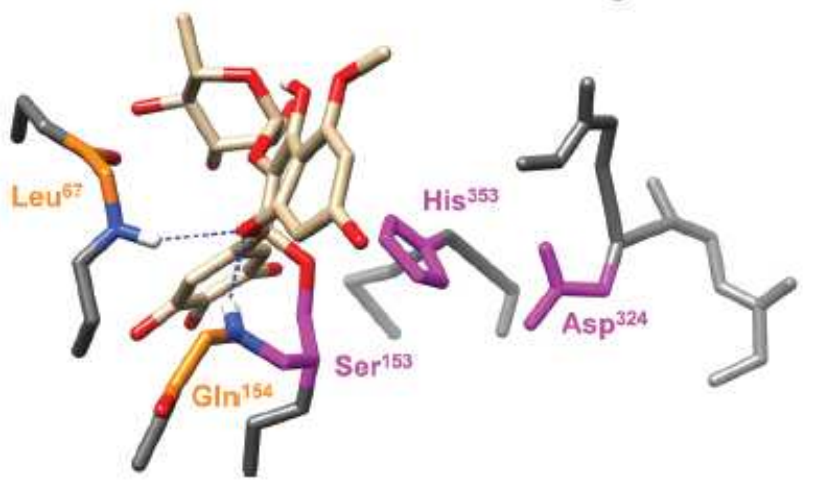


604 Table 1. $\mathrm{a}_{\mathrm{I} 50}$ and $\mathrm{t}_{1 / 2}$ values of SAPRF4 on DGL, HPL and LipY.

605

\begin{tabular}{ccc}
\hline Lipases & $\mathrm{a}_{150}(\mu \mathrm{g})$ & $\mathrm{t}_{1 / 2}(\min )$ \\
\hline DGL & 7 & 0.4 \\
HPL & 82 & 0.6 \\
LipY & 5 & 0.1 \\
\hline
\end{tabular}

606

607 SAPRF4 amount (a $\mathrm{a}_{\mathrm{I}}$ ) used for the determination of $\mathrm{t}_{1 / 2}$ was $10 \mu \mathrm{g}, 200 \mu \mathrm{g}$ and $10 \mu \mathrm{g}$ for 608 DGL, HPL and LipY, respectively. Results are expressed as mean values of at least three 609 independent assays (CV \% $<5.0 \%)$.

610 